Proceeding Series of the Brazilian Society of Computational and Applied Mathematics

\title{
Simulações de problemas em magneto-hidrodinâmica usando o código FLASH
}

Edgard de F. D. Evangelista ${ }^{1}$

Bolsista do CNPq-Brasil.

Instituto Nacional de Pesquisas Espaciais, CEA, São José dos Campos, SP

Margarete O. Domingues ${ }^{2}$

Instituto Nacional de Pesquisas Espaciais, LAC, São José dos Campos, SP

Odim Mendes Jr. ${ }^{3}$

Instituto Nacional de Pesquisas Espaciais, CEA, São José dos Campos, SP

Oswaldo D. Miranda ${ }^{4}$

Instituto Nacional de Pesquisas Espaciais, CEA, São José dos Campos, SP

Resumo. A magneto-hidrodinâmica (MHD) surge da combinação das equações da hidrodinâmica e do eletromagnetismo. Existem diversos códigos que lidam com MHD, como o FLASH e o Athena. O objetivo é a avaliação do FLASH por meio de comparações quando operando sob diferentes modos. O Athena encontra-se em fase de implementação.

Palavras-chave. Fluidodinâmica, Plasma, Modelagem, Magneto-hidrodinâmica, código FLASH

\section{Introdução e Conceitos Teóricos}

A MHD consiste basicamente no estudo de fluidos compressíveis e condutores de eletricidade sob a influência de campos magnéticos. As equações que governam o comportamento de tais sistemas são obtidas através da combinação das equações de Maxwell do eletromagnetismo com as equações de Euler da hidrodinâmica. Nesse contexto, há a MHD ideal, onde a viscosidade e a resistividade são desprezadas; a MHD não-ideal é o caso onde tais condições não podem ser mantidas.

O formalismo da MHD é de grande interesse para diversas áreas, destacando-se a geofísica espacial e a astrofísica, uma vez que a maior parte da matéria bariônica do universo é formada de plasma. No entanto, o sistema de equações da MHD é matematicamente complexo e suas soluções requerem a utilização de métodos numéricos e programas

\footnotetext{
${ }^{1}$ edgard.evangelista@inpe.br

${ }^{2}$ margarete.domingues@inpe.br

${ }^{3}$ odim.mendes@inpe.br

${ }^{4}$ oswaldo.miranda@inpe.br
} 
computacionais, tais como o FLASH [3] e o Athena [12]. No presente artigo é utilizado o código FLASH para gerar as simulações de dois problemas clássicos em MHD: o tubo de choque [2] e o problema de Orszag-Tang (OT) [9]. O objetivo dos testes com o tubo de choque é a análise do desempenho do FLASH quando usado com diferentes esquemas numéricos e condições. Já o resultado para o problema de OT será comparado aos encontrados na literatura.

A seguir são apresentados os fundamentos teóricos necessários; em seguida o propósito e a metodologia são discutidos; depois, as próximas seções trazem, nessa ordem, os resultados e a discussão e os comentários finais.

\subsection{Magneto-hidrodinâmica}

De forma geral, o sistema de equações da MHD é construído através da união entre as equações de Euler (que descrevem a dinâmica dos fluidos) e as equações de Maxwell do eletromagnetismo. É importante especificar que as equações de Maxwell impõem um vínculo ao modelo. Tal vínculo é representado pela lei de Gauss do campo magnético e enunciado, em sua forma diferencial, na forma: o divergente do vetor campo magnético é nulo em todos os pontos do domínio considerado.

Seguindo o esquema mostrado em [11], o sistema de equações que compõe o modelo MHD ideal para três dimensões é caracterizado por:

$$
\begin{aligned}
& \frac{\partial \rho}{\partial t}+\nabla \cdot(\rho \mathbf{u})=0 \\
& \frac{\partial \rho \mathbf{u}}{\partial t}+\nabla \cdot\left(\rho \mathbf{u u}+\left(p+\frac{\mathbf{B B}}{2 \mu_{0}}\right) \mathbf{I}-\frac{\mathbf{B B}}{\mu_{0}}\right)=-\frac{1}{\mu_{0}} \mathbf{B} \nabla \cdot \mathbf{B} ; \\
& \frac{\partial \mathbf{B}}{\partial t}+\nabla \cdot(\mathbf{u B}-\mathbf{B u})=-\mathbf{u} \nabla \cdot \mathbf{B} \\
& \frac{\partial E}{\partial t}+\nabla \cdot\left[\left(E+p+\frac{\mathbf{B B}}{2 \mu_{0}}\right) \mathbf{u}-\frac{1}{\mu_{0}}(\mathbf{u} \cdot \mathbf{B}) \mathbf{B}\right]=-\frac{1}{\mu_{0}}(\mathbf{u} \cdot \mathbf{B}) \nabla \cdot \mathbf{B},
\end{aligned}
$$

em que $\rho, E, p, \mu_{0}, \mathbf{u}$ e $\mathbf{B}$ são, respectivamente, a densidade, a energia, a pressão, a permeabilidade magnética do vácuo, a velocidade e o campo magnético. Além disso, I é a matriz unidade $3 \times 3$.

Note que nas Eqs. 1 os termos $\nabla \cdot \mathbf{B}$ foram mantidos, embora sejam analiticamente nulos. Isso ocorre porque, do ponto de vista do cálculo numérico, é difícil assegurar que tal quantidade seja exatamente zero, de forma que nos esquemas computacionais o termo deve ser mantido. Em [1] é descrito um método no qual o vínculo da divergência nula é adicionado às equações por meio de um multiplicador de Lagrange generalizado. Além disso, como será visto, o FLASH tem algumas maneiras de lidar com o problema.

\subsection{Volumes finitos e fluxos numéricos}

O método dos volumes finitos é uma técnica para a solução de equações diferenciais parciais, especialmente aquelas que representam leis físicas de conservação. Nesse esquema, 
os valores das grandezas físicas são calculados em regiões discretas nas quais o domínio é dividido. A denominação "volume finito" se refere a tais regiões [7].

Nesse método as integrais de volume que possuem um divergente no integrando e que surgem a partir da solução das equações diferenciais são transformadas em integrais de superfície por meio do teorema de Gauss. Essas integrais são então interpretadas como fluxos através das superfícies de cada volume no qual o domínio é dividido.

Uma característica importante do método dos volumes finitos é o fato de os fluxos numéricos serem conservativos, isto é, o fluxo é conservado entre duas células adjacentes. Tal propriedade torna o método apropriado para modelagens nas quais os fluxos e as leis de conservação têm papel importante, como na dinâmica dos fluidos e nas transferências de massa e energia.

Há diversas maneiras conhecidas de se calcular os fluxos numéricos mencionados anteriormente. Elas variam em precisão numérica e em custo computacional. Por exemplo, no FLASH o método padrão é o Roe [6]. Entretando, em alguns casos a utilização de alternativas é útil, em especial em algumas situações de plasma espacial. Nesse contexto, há outros fluxos numéricos como o HLL [4], HLLC [8] e HLLD [10]; um modelo híbrido, que combina o esquema de Roe com o HLLD também é utilizado frequentemente.

\subsection{Métodos split e unsplit}

O método split consiste em uma técnica para a solução de equações diferenciais. Seja, por exemplo, o problema de Cauchy

$$
\left\{\begin{array}{c}
\frac{\mathrm{d} y}{\mathrm{~d} t}=A(t) y+g(t), t \in(0, t] \\
y(0)=y_{0},
\end{array}\right.
$$

em que $y_{0} \in \mathbb{R}^{d}, A(t) \in \mathbb{R}^{d \times d}$ and $g(t) \in \mathbb{R}^{d}$ são funções matriciais dependentes do tempo. Apesar da aparente simplicidade, esse tipo de problema geralmente demanda métodos numéricos mais sofisticados para sua solução. Dentre os métodos existentes destaca-se o split, cuja ideia básica é dividir a Eq. 2 em dois subproblemas e então resolver o sistema resultante ciclicamente, usando a solução da iteração atual como condição inicial da iteração seguinte [5].

Particularmente, o FLASH utiliza o eight-wave, proposto por [11]. Esse esquema é baseado em um método de volume finito, onde cada célula é localizada pelas coordenadas de seu centro. O algoritmo usa um splitting direcional para evoluir as equações da MHD, fazendo uma varredura em cada direção espacial para evoluir as variáveis físicas de um nível temporal para outro. Depois que todas as varreduras são completadas, o algoritmo aplica a condição $\nabla \cdot \mathbf{B}=0$ por meio de um método difusivo chamado "método de truncamentoerro".

Além do esquema descrito acima, o FLASH utiliza também, como alternativa, o chamado unsplit staggered mesh algorithm (USM), que resolve as equações para o caso de MHD ideal e não-ideal em múltiplas dimensões. Este baseia-se no método de Godunov de alta ordem combinado ao esquema constrained transport para lidar com o vínculo $\nabla \cdot \mathbf{B}=0$. 
De acordo com [13], a formulação staggered mesh consiste em se definir as componentes do campo magnético nas interfaces das células, os campos elétricos nas arestas e todas as variáveis termodinâmicas nos centros das mesmas. Uma das vantagens mais interessantes desse método é que as componentes do campo magnético satisfazem localmente à condição de divergência nula.

\section{Propósito e Métodos}

São utilizados os códigos FLASH e Athena para a geração e comparação de simulações dos problemas do tubo de choque e de OT, ambos dentro do formalismo da MHD ideal.

O problema do tubo de choque em uma dimensão é empregado para se comparar os resultados gerados pelo FLASH quando usando os fluxos numéricos HLL, HLLC, HLLD e Roe, em que cada um deles foi executado com os métodos split e unsplit. Além disso são testados os casos com e sem a correção para o divergente do campo magnético B. A Tabela 1 mostra os valores iniciais das grandezas para o problema em questão. É utilizada uma resolução de $512 \times 512$, com o número de Courant, Friedrichs e Lewy (CFL) igual a 0,8 , coeficiente de expansão adiabática $\gamma$ igual a $5 / 3, t_{\max }=0,1 \mathrm{~s}$, em um domínio $[-0.5,0.5] \times[-0.5,0.5]$.

Tabela 1: Configuração inicial para o problema do tubo de choque [2].

\begin{tabular}{|c|c|c|c|c|c|c|c|c|}
\hline & $\rho$ & $p$ & $v_{x}$ & $v_{y}$ & $v_{z}$ & $B_{x}$ & $B_{y}$ & $B_{z}$ \\
\hline$x \leq 0$ & 1,08 & 0,95 & 1,20 & 0,01 & 0,05 & $1,00 / \sqrt{\pi}$ & $1,80 / \sqrt{\pi}$ & $2,00 / \sqrt{2 \pi}$ \\
\hline$x>0$ & 1,00 & 1,00 & 0,00 & 0,00 & 0,00 & $1,00 / \sqrt{\pi}$ & $2,00 / \sqrt{\pi}$ & $2,00 / \sqrt{2 \pi}$ \\
\hline
\end{tabular}

Por outro lado, é utilizado o FLASH para a simulação do problema de OT em duas dimensões. Nesse caso são definidas as condições: fluxo HLLD, resolução de $256 \times 256$, $t_{\max }=0,5 \mathrm{~s}, \gamma=5 / 3$ e CFL $=0,8$. A Tabela 2 apresenta a condição inicial das grandezas utilizadas no problema.

Tabela 2: Configuração inicial para o problema de OT [13].

\begin{tabular}{|c|c|c|c|c|c|c|c|}
\hline$\rho$ & $p$ & $v_{x}$ & $v_{y}$ & $v_{z}$ & $B_{x}$ & $B_{y}$ & $B_{z}$ \\
\hline$\gamma^{2}$ & $\gamma$ & $-\sin (2 \pi y)$ & $\sin (2 \pi x)$ & 0,0 & $-\sin (2 \pi y)$ & $\sin (4 \pi x)$ & 0,0 \\
\hline
\end{tabular}

Em todos os casos é utilizado o sistema de unidades centímetro-grama-segundo (CGS).

\section{Resultados e Discussão}

Figuras 1(a)-2(b) mostram os perfis de densidade do fluido em $t=0,1 \mathrm{~s}$. Da esquerda para a direita e de cima para baixo, tem-se os resultados usando Roe, HLL, HLLC e 
HLLD. Em cada caso o código é testado com split e unsplit, com e sem a restrição $\nabla$. $\mathbf{B}=0$. Comparando os resultados à curva de referência, como discutido em [2], nota-se concordância em todos os casos, exceto quando usando unsplit sem a correção da condição $\nabla \cdot \mathbf{B}=0$. Isso indica que o FLASH é estável e artefatos numéricos não apareceram neste caso trabalhando com todas as opções de fluxos e sob os esquemas split e unsplit. No entanto, mesmo neste caso simples iniciado com uma condição de Riemman 1D o método unsplit necessita da correção da condição de divergência nula de $\mathbf{B}$. Utilizando-se condições iniciais mais sofisticadas sem essa correção da condição de divergência nula de B mesmo o método split apresenta artefatos numéricos indesejáveis na solução.

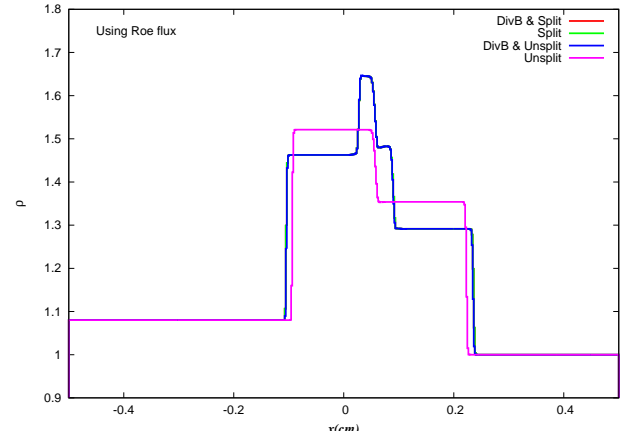

(a) Roe

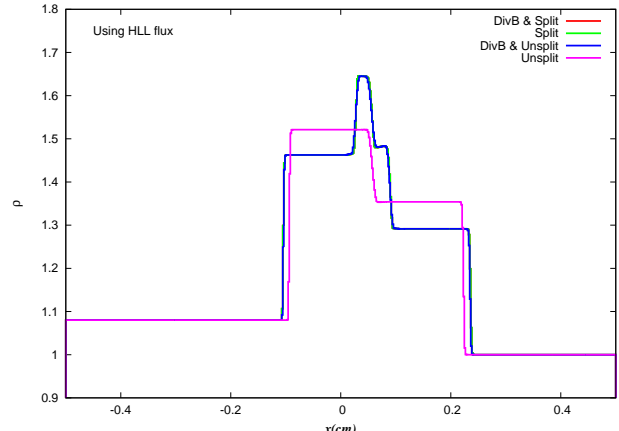

(b) HLL

Figura 1: Problema do tubo de choque em $t=0,1 \mathrm{~s}$ obtido com o FLASH e usando os fluxos Roe e HLL. Considera-se MHD ideal.

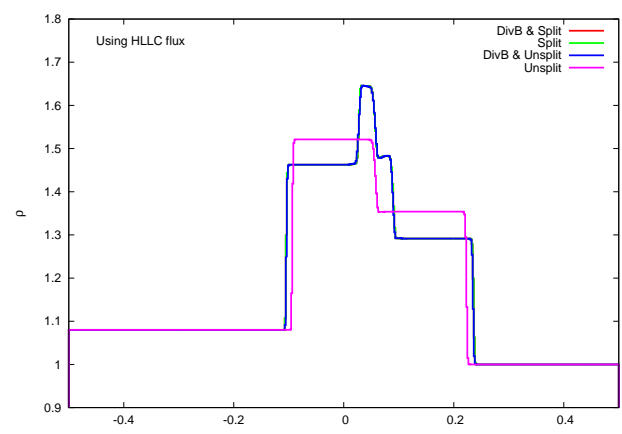

(a) HLLC

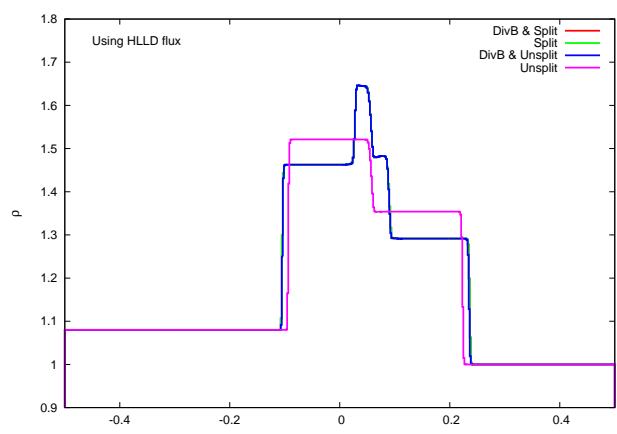

(b) HLLD

Figura 2: Ídem, usando HLLC e HLLD.

Figura 3 mostra o perfil de densidade para o problema de OT no instante $t=0,5$ s. É considerada a MHD ideal, com condições especificadas na Seção 2. Comparando esse perfil aos resultados mostrados, por exemplo, em [13], nota-se que a configuração apresenta concordância, tornando o resultado uma referência importante para o teste de outros códigos.

Os autores têm utilizado o FLASH rotineiramente na geração de diversas simulações, enquanto o Athena encontra-se atualmente em fase de implementação e testes. Portanto, 
seguindo o raciocínio acima, resultados como o da Fig. 3 serão comparados aos gerados pelo Athena. Essas comparações darão informações a respeito do funcionamento do Athena, onde será interessante também avaliar se vínculos de natureza física, como por exemplo a divergência nula de $\mathbf{B}$, são tratados de forma adequada pelo código.

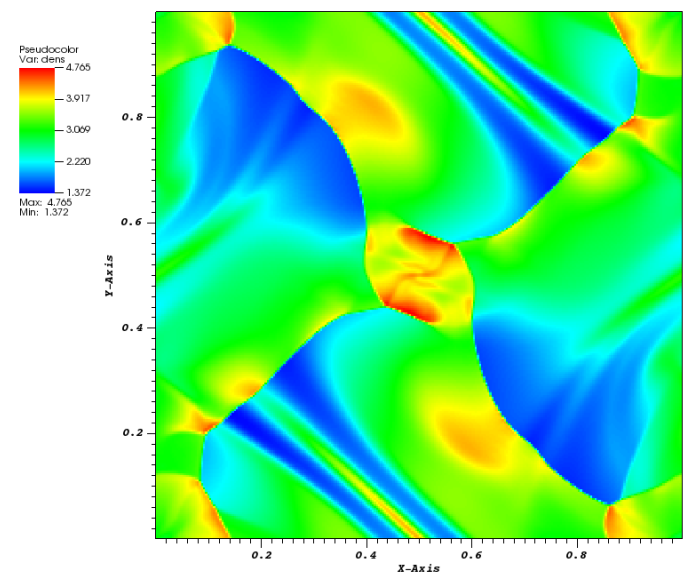

Figura 3: Perfil da densidade em $t=0,5 \mathrm{~s}$ para o problema de OT usando FLASH. Considera-se MHD ideal.

\section{Comentários finais}

No presente artigo são comparados os resultados gerados pelo código FLASH com o uso dos fluxos Roe, HLL, HLLC e HLLD, operando nos esquemas split e unsplit. Além disso são considerados os casos onde o vínculo $\nabla \cdot \mathbf{B}=0$ é ou não utilizado. Percebe-se que há discrepância apenas nos casos onde é usado unsplit sem a imposição de $\nabla \cdot \mathbf{B}=0$; os outros casos são essenciamente coincidentes.

A fim de realizar testes com o código Athena, o qual encontra-se em fase de implementação, os autores utilizam um problema clássico em MHD chamado problema de Orszag-Tang, cujas simulação gerada pelo FLASH é comparada com resultados encontrados em [13]. Dado que o resultado gerado é confiável, será utilizado para validar as futuras simulações do Athena.

Em trabalhos futuros serão mostrados os testes com o código Athena, onde seus resultados serão comparados aos gerados por outros códigos como o FLASH. Oportunamente, haverá comparação também com resultados encontrados na literatura. Tais testes terão como objetivo investigar o funcionamento dos códigos e aprimorar seu desempenho e a confiabilidade.

\section{Agradecimentos}

Os autores agradecem ao CNPq pelo apoio financeiro, por meio do processo número $158967 / 2014-3$. 


\section{Referências}

[1] A. Dedner, F. Kenn, D. Kröner, T. Schnitzer and M. wesemberg, Hyperbolic divergence cleaning for the MHD equations, J. Comp. Phys., vol. 175, 645-673, (2002).

[2] M. O. Domingues, A. K. F. Gomes, S. M. Gomes, O. mendes. B. D. Pierro and K. Schneider, Extended generalized lagrangian multipliers for magnetohydrodynamics using adaptative multiresolution methods, ESAIM:Proceedings, vol. 43, 95-107, (2013).

[3] A. Dubey, L. B. Reid e R. Fisher, Introduction to FLASH 3.0 with application to supersonic turbulence, Phys. Scr., vol. T132, 014046, (2008).

[4] B. Einfeld, C. D. Munz, P. L. Roe and B. Sjögreen, On Godunov-type methods near low densities, J. Comp. Phys., vol. 92, 273-295, (1991).

[5] I. Faragó, A. Havasi and R. Horváth, On the Order of Operator Splitting Methods for Time-dependent Linear Systems of Differential Equations, Int. J. Num. Anal. Mod., vol. 2, 142-154, (2011).

[6] A. Harten, High resolution schemes for hyperbolic conservation laws, J. Comp. Phys., vol. 49, 357-393, (1983).

[7] R. J. LeVeque, Finite Volume Methods for Hyperbolic Problems, Cambridge University Press, Cambridge, (2002).

[8] S. Li, An HLLC Riemann solver for magneto-hydrodynamics, J. Comp. Phys., vol. 203, 344-357, (2005).

[9] A. Mignone, P. Tzeferacos and G. Bodo, High-order conservative finite difference GLM-MHD schemes for cell-centered MHD, J. Comp. Phys., vol. 229, 5896-5920, (2010).

[10] T. Miyoshi and K. Kusano, A multi-state HLL approximate Riemann solver for ideal magnetohydrodynamics, J. Comp. Phys., vol. 208, 315-344, (2005).

[11] K. G. Powell, P. L. Roe, T. J. Linde, T. I. Gombosi and D. L. De Zeeuw, A SolutionAdaptative Upwind Scheme for Ideal Magnetohydrodynamics, J. Comp. Phys., vol. 154, 284-309, (1999).

[12] J. M. Stone, T. A. Gardiner, P. Teuben, J. F. Hawley and J. B.Simon, Athena: a new code for astrophysical MHD, Astrophys. J. Suppl. S., vol. 178, 137-177, (2008).

[13] J. Vides, E. Audit, H. Guillard and B. Nkonga, Divergence-free MHD Simulations with the Heracles Code, ESAIM: Proceedins, vol. 43, 180-194, (2013). 